\title{
Decelerated Liquid Dynamics Induced by Component-Dependent Supercooling in Hydrogen and Deuterium Quantum Mixtures
}

\section{$\operatorname{AUTHOR}(S):$}

Yamaoka, Shutaro; Kim, Hyeon-Deuk

\section{CITATION:}

Yamaoka, Shutaro ... [et al]. Decelerated Liquid Dynamics Induced by ComponentDependent Supercooling in Hydrogen and Deuterium Quantum Mixtures. The journal of physical chemistry letters 2020, 11(10): 4186-4192

\section{ISSUE DATE:}

2020-05-21

URL:

http://hdl.handle.net/2433/252779

\section{RIGHT:}

This document is the Accepted Manuscript version of a Published Work that appeared in final form in The journal of physical chemistry letters, copyright $\odot$ American Chemical Society after peer review and technical editing by the publisher. To access the final edited and published work see https://doi.org/10.1021/acs.jpclett.0c00801; The full-text file will be made open to the public on 6 May 2021 in accordance with publisher's 'Terms and Conditions for SelfArchiving'.; この論文は出版社版でありません。引用の際には出版社版をご確認ご利用ください。; This is not the published version. Please cite only the published version. 


\title{
Decelerated Liquid Dynamics Induced by Component-Dependent Supercooling in \\ Hydrogen and Deuterium Quantum Mixtures
}

\author{
Shutaro Yamaoka and Kim Hyeon-Deuk* \\ Department of Chemistry, Kyoto University, Kyoto, 606-8502, Japan \\ E-mail: kim@kuchem.kyoto-u.ac.jp,Phone:+81-75-753-4021
}

${ }^{*}$ To whom correspondence should be addressed 


\begin{abstract}
Isotopic mixtures of $p-\mathrm{H}_{2}$ and $o-\mathrm{D}_{2}$ molecules have been an attractive binary system because it includes two kinds of purely isotopic molecules which possess the same electronic potential but the twice different mass inducing differently pronounced nuclear quantum effects(NQEs). Accessing details of structures and dynamics in such quantum mixtures combining complex molecular dynamics with NQEs of different strength remains a challenging problem. Taking the advantage of the non-empirical molecular dynamics method which describes $p-\mathrm{H}_{2}$ and $o-\mathrm{D}_{2}$ molecules, we found that the liquid dynamics slows down at a specific mixing ratio, which can be connected to the observed anomalous slowdown of crystallization in the quantum mixtures. We attributed the decelerated dynamics to the component-dependent supercooling of $p-\mathrm{H}_{2}$ taking place in the mixtures, demonstrating that there is an optimal mixing ratio to hinder crystallization. The obtained physical insights will help in experimentally controlling and achieving unknown quantum mixtures including superfluid.
\end{abstract}

\title{
TOC Graphic.
}

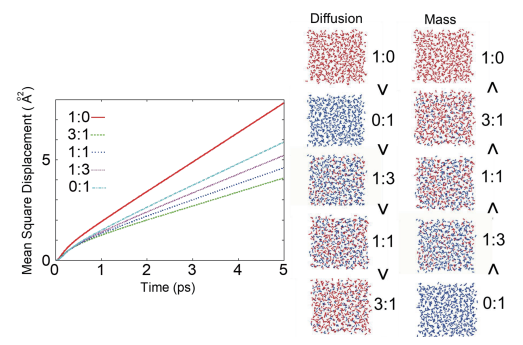


Knowledge of mechanisms that governs liquid structure and dynamics in mixtures is important to elucidate the basic nature of solution including crystallization and chemical reaction. Binary mixtures generally exhibit properties that differ fundamentally from their pure liquids. Binary systems composed of model particles have been employed to investigate crystallization and glassy behaviors in mixtures. ${ }^{1-9}$ The correlation between compositions and crystallization kinetics was found that the crystal growth becomes slow with a specific mixing rate in the mixtures of differently sized model particles. ${ }^{8}$ Water mixtures are especially important and have been calculated and observed widely in normal water solutions, ${ }^{10-31}$ hydrogels, ${ }^{32-34}$ and supercooled and critical water solutions. ${ }^{5,6,35-38}$ Notably, the minima of the self-diffusion coefficients were found in the mixtures of water and organic molecules experimentally ${ }^{39,40}$ and computationally; ${ }^{28,29,41-47}$ the self-diffusion coefficients decrease, pass through the minima, and then increase by mixing another component. Mixtures of $p-\mathrm{H}_{2}$ and $o-\mathrm{D}_{2}$ have been an ideal and attractive binary liquid because they purely includes two kinds of isotope molecules which experience the same electronic potential and thus the negligibly small mixing enthalpy but the twice different mass inducing differently pronounced nuclear quantum effects(NQEs) and different thermodynamic properties. ${ }^{48-52}$ However, accessing structures and dynamics in such quantum mixture which combines complex molecular dynamics possessing different NQEs and thermodynamic properties still remains a challenge. The presence of $o-\mathrm{D}_{2}$ modifies self-dynamics of $p-\mathrm{H}_{2}$ in a highly nontrivial way:e.g. Although the slowdown in the crystallization was observed in their mixtures, an understanding of how a composition ratio decelerates crystallization kinetics in the isotope mixtures is still lacking. ${ }^{49,50,53}$

While the semiquantum methods based on the imaginary-time path-integral theory were proposed, ${ }^{54,55}$ we recently developed the non-empirical ab initio simulation method of nuclear and electron wave packet molecular dynamics (NEWPMD) where the important NQEs such as nuclear delocalization and zero-point energy were non-perturbatively taken into account. ${ }^{56-62}$ The NEWPMD method has successfully given the structural and dynamical 
properties which are close to the experimental observations in the condensed $p-\mathrm{H}_{2}$ and $o-\mathrm{D}_{2}$ systems reflecting the proper isotope-dependent NQEs. Specifically, the NEWPMD method has reproduced the radial distribution functions and self-diffusion coefficients in the $p-\mathrm{H}_{2}$ and $o-\mathrm{D}_{2}$ vapor-pressure liquids including their anomalous temperature dependence, ${ }^{57,58,62}$ the hexagonal close-packed structures, librational frequency and collective phonon modes in the $p-\mathrm{H}_{2}$ vapor-pressure solids, ${ }^{59}$ the freezing temperature indicated by the stable $p-\mathrm{H}_{2}$ vapor-pressure solid phase at $13 \mathrm{~K}$ and the stable $p$ - $\mathrm{H}_{2}$ vapor-pressure liquid phase at $14 \mathrm{~K}$ in addition to the metastable supercooled phase with the lower density even below the freezing temperature, ${ }^{59,60}$ the boson peak around $20 \mathrm{~cm}^{-1}$ in the supercooled $p-\mathrm{H}_{2}$ liquids analogous to the low-frequency boson peaks observed in various glasses and supercooled liquids, ${ }^{60}$ the intramolecular bond length and vibrational frequencies of $p-\mathrm{H}_{2}$ and $o-\mathrm{D}_{2}$ molecules including the discrete frequency jump due to the phase transition from the $p-\mathrm{H}_{2}$ vapor-pressure liquid to solid, ${ }^{58,59}$ and the thermal conductivity directly obtained through Fouriers law in the non-equilibrium $p$ - $\mathrm{H}_{2}$ liquids under heat conduction. ${ }^{61}$ The above facts ensure that the NEWPMD simulations reasonably sit in the experimental thermodynamic region. If the NEWPMD simulations sat in an anomalous thermodynamic region, we could never get the above reasonable agreements in the various thermodynamic situations. The NEWPMD method is thus distinguished from most of the previous path-integral approaches for a condensed phase which require an equilibrium partition function as well as an intermolecular model potential including empirical parameters like the Silvera-Goldman potential without intramolecular degrees of freedom. ${ }^{63}$ In this Letter, taking the advantage of the NEWPMD method, we investigate liquid structure and dynamics which are intrinsic to the quantum isotopic mixtures of $p-\mathrm{H}_{2}$ and $o-\mathrm{D}_{2}$, and report the anomalous correlations between the mixing ratio and liquid dynamics, which will help in experimentally controlling and achieving unknown quantum mixtures including superfluid. For simplicity, $p-\mathrm{H}_{2}$ and $o-\mathrm{D}_{2}$ will be simply described as $\mathrm{H}_{2}$ and $\mathrm{D}_{2}$ throughout this Letter.

We adopted the NEWPMD method which expresses each nucleus by a floating and thawed 
Gaussian nuclear wave packet(NWP) using the time-dependent Hartree approach, and each electron by an electron wave packet(EWP) to follow the perfect-pairing valence bond theory that appropriately treats the Pauli exclusion energy. ${ }^{57,58}$ All the ab initio intramolecular and intermolecular interactions as diatomic molecules were non-empirically derived via the variational principle with the time-dependent wave function for $\mathrm{H}_{2}$ and $\mathrm{D}_{2}$ dimers nonperturbatively treating the NWPs and EWPs. ${ }^{56}$ The electronic part of the NEWPMD program was not modified for $\mathrm{H}_{2}$ and $\mathrm{D}_{2}$ while the atomic mass of each molecule changes depending on the mixing isotopes, 1.00794 for $\mathrm{H}_{2}$ and 2.01410 for $\mathrm{D}_{2}$, respectively. We simulated 640 molecules in a three-dimensional(3D) cubic simulation box with a periodic boundary condition at the three temperatures, $30 \mathrm{~K}, 25 \mathrm{~K}$, and $18 \mathrm{~K}$. The five mixing ratios were set as $\mathrm{D}_{2}$ ratios of $0 \%, 25 \%, 50 \%, 75 \%$, and $100 \%$ which we will call the pure $\mathrm{H}_{2}$ liquid or 1:0 mixture, 3:1 mixture, 1:1 mixture, 1:3 mixture, and pure $\mathrm{D}_{2}$ liquid or 0:1 mixture, respectively. We set the molar volume as $28.08 \times 10^{-6} \mathrm{~m}^{3} / \mathrm{mol}$ at $30 \mathrm{~K}$ and $25.17 \times 10^{-6} \mathrm{~m}^{3} / \mathrm{mol}$ at $25 \mathrm{~K}$ which are close to the vapor-pressure molar volume for $\mathrm{D}_{2}$ at the corresponding temperature. ${ }^{48,64}$ The molar volume $23.20 \times 10^{-6} \mathrm{~m}^{3} / \mathrm{mol}$ at $18 \mathrm{~K}$ was set by extrapolating the vapor-pressure line for $\mathrm{D}_{2}$ down to $18 \mathrm{~K}$. It is thus much smaller than the vapor-pressure molar volume for $\mathrm{H}_{2}$, i.e. $27.39 \times 10^{-6} \mathrm{~m}^{3} / \mathrm{mol}$ at $18 \mathrm{~K}$. We matched the thermodynamic condition regardless of the $\mathrm{D}_{2}$ mixing ratio in order to purely compare and elucidate the isotope mixing effects. We adopted the hexagonal-close-packed crystal structures with random orientation as an initial structure, and performed cooling and equilibration runs by the velocity scaling thermostat and then the Berendsen thermostat for several hundreds picoseconds at the aimed temperatures. ${ }^{57,58}$ After these careful cooling and equilibration runs, we carried out the NVE (microcanonical) simulations for several hundreds picoseconds, and obtained the reasonable temperatures close to the aimed temperatures.(Table S1) The averages of the nuclear kinetic energy and remaining potential energies are also shown in Fig.S1. The snapshot views of the $3 \mathrm{D}$ cubic simulation boxes containing 640 molecules of $\mathrm{H}_{2}$ and $\mathrm{D}_{2}$ are shown in Fig.S2. 
A liquid structure can be characterized by a radial distribution function(RDF) as a function of distance between centers of two NWPs. The whole shapes of the calculated RDFs for the $\mathrm{H}_{2}-\mathrm{D}_{2}$ mixtures are given in Fig.S3. The RDFs exhibit little difference regardless of the temperature and the $\mathrm{D}_{2}$ ratio. The electronic potentials created by the EWPs are identical for $\mathrm{H}_{2}$ and $\mathrm{D}_{2}$, leading to the similar RDFs under the same thermodynamic conditions although much lower temperature could induce a difference. ${ }^{62}$ The maximum difference of $0.0149 \AA$ between the H-H and D-D bond length does not induce any significant difference in the present RDFs. (See Fig.S4) Figure S5 also shows that the distributions of the intermolecular angle $\theta$ between two molecules are almost uniform in any case. $\mathrm{H}_{2}$ and $\mathrm{D}_{2}$ are fully liquidized and there is no static orientational correlation between molecules in the whole system. ${ }^{65}$
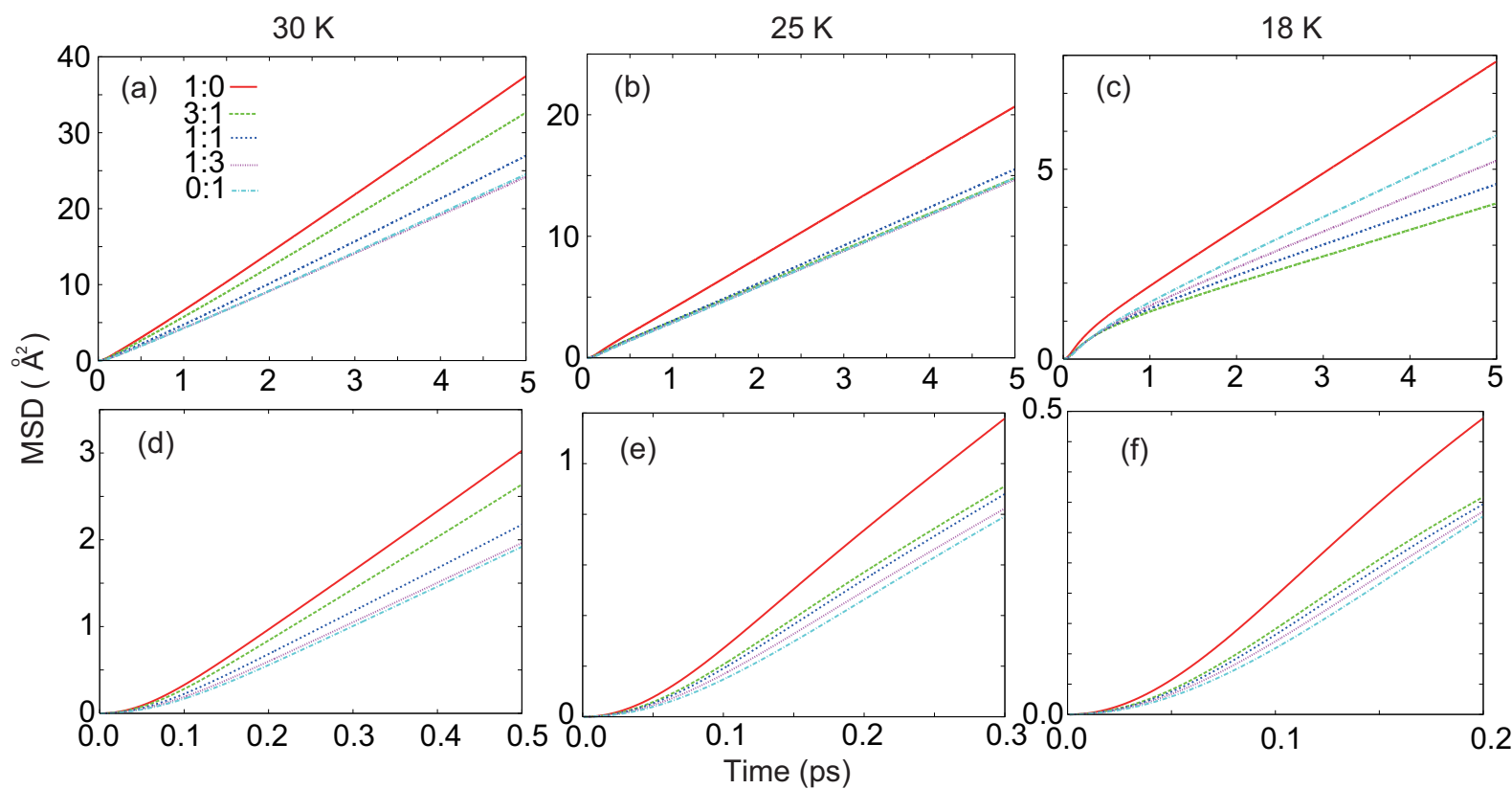

Figure 1: MSDs at (a) $30 \mathrm{~K}$, (b) $25 \mathrm{~K}$, and (c) $18 \mathrm{~K}$. The corresponding MSDs in the initial time region are magnified in $(\mathrm{d})-(\mathrm{f})$.

A mean square displacement(MSD) characterizes liquid dynamics including kinetic motion inside a solvation shell and long-range diffusion out of a shell. ${ }^{60,62,66,67}$ The MSDs of the $\mathrm{H}_{2}-\mathrm{D}_{2}$ mixtures at the different temperatures are shown in Figs.1(a)-(c). The pure $\mathrm{H}_{2}$ liquid exhibits the fastest dynamics at any temperature because of the lightest mass. At 
the highest temperature of $30 \mathrm{~K}$, the MSD decreases with the $\mathrm{D}_{2}$ ratio; the higher the $\mathrm{D}_{2}$ rate is, the slower the liquid dynamics becomes simply following the mass order. However, the MSDs at the lower temperatures exhibit the qualitatively different order. All the MSDs other than the pure $\mathrm{H}_{2}$ MSD are almost overlapped at $25 \mathrm{~K}$, and the MSDs at $18 \mathrm{~K}$ are in the order of the 1:0, 0:1, 1:3,1:1, and 3:1 mixtures, which is surprising considering the fact that the liquid and molecular structures of these mixtures are almost similar and that the temperature and density are the same. (See Figs.S3, S4 and S5)

These anomalous orders of the MSDs in spite of the little difference in the structures can be explained by the component-dependent supercooling: $\mathrm{H}_{2}$ molecules are effectively supercooled in the lower-temperature mixtures. The current molar volume set close to the vapor-pressure line for $\mathrm{D}_{2}$ is much smaller than the vapor-pressure molar volume for $\mathrm{H}_{2}$ : e.g. the molar volume set as $23.20 \times 10^{-6} \mathrm{~m}^{3} / \mathrm{mol}$ at $18 \mathrm{~K}$ is even smaller than the vapor-pressure molar volume for $\mathrm{H}_{2}$ at $10 \mathrm{~K}, 25.08 \times 10^{-6} \mathrm{~m}^{3} / \mathrm{mol}$. Therefore, $\mathrm{H}_{2}$ molecules can be effectively supercooled due to the high density even at the temperature higher than the freezing point of $\mathrm{H}_{2}, 13.8 \mathrm{~K}$. The supercooling as well as the solidification can be induced not only by lowering the temperature but also by increasing the density. In fact, the present pure $\mathrm{H}_{2}$ liquid at $18 \mathrm{~K}$ clearly exhibit the behavior of the supercooling as will be demonstrated below as the nonlinear MSDs in the initial stage and the broad peak around $20 \mathrm{~cm}^{-1}$ analogous to the boson peaks. ${ }^{60,62}$ The supercooled $\mathrm{H}_{2}$ can be a source to hinder the diffusive dynamics in the mixtures, leading to the anomalous orders of the MSDs at the lower temperature.

The nonlinear profiles of the MSDs appearing within 0.5 ps in Fig.1(c) support this supercooling picture because the nonlinear MSDs appeared when the pure $\mathrm{H}_{2}$ and $\mathrm{D}_{2}$ liquids became supercooled below the freezing temperature, ${ }^{60,62}$ and such nonlinear MSDs finally lead to the local- $\beta$ relaxation observed in the various glasses and supercooled liquids. ${ }^{66-68}$ We note that, since $18 \mathrm{~K}$ is slightly lower than the freezing point of $\mathrm{D}_{2}(18.7 \mathrm{~K})$, the initial MSD of the pure $\mathrm{D}_{2}$ liquid is also nonlinear. However, the extent of the supercooling is more significant for $\mathrm{H}_{2}$ in the current cramped molar volume. As a result, the 3:1 mixture 
which includes most $\mathrm{H}_{2}$ among the $\mathrm{H}_{2}-\mathrm{D}_{2}$ mixtures exhibits the smallest MSD at $18 \mathrm{~K}$, being conflict with the mass order. The liquid mixture at $25 \mathrm{~K}$ is just in an intermediate state between $18 \mathrm{~K}$ and $30 \mathrm{~K}$. The initial MSDs shown in Figs.1(d)-(f) correspond to an initial kinetic motion confined in a first solvation cage, and should be much less influenced by the supercooling. In fact, the initial MSDs are simply larger with the lighter liquid mass regardless of the temperature; their order is similar to the order of the long-time MSDs at 30 K because of the absence of the supercooling effect.(Compare Fig.1(a) and Figs.1(d)-1(f))

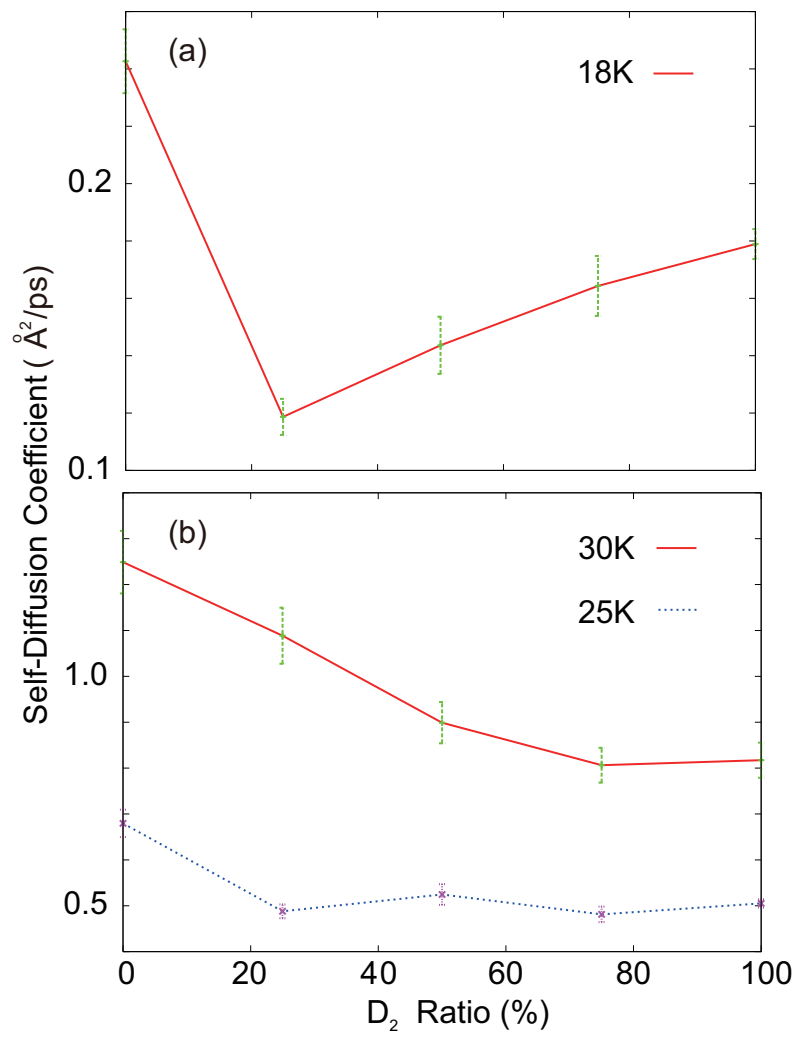

Figure 2: Self-diffusion coefficients at the different temperatures as a function of the $\mathrm{D}_{2}$ ratio.

The self-diffusion coefficients estimated from the MSDs are summarized in Fig.2. The self-diffusion coefficient of the pure $\mathrm{D}_{2}$ liquid at $25 \mathrm{~K}, 0.51 \AA^{2} / \mathrm{ps}$, is reasonable taking into account a fact that the current diffusion coefficient would increase by approximately $20 \%$ in an infinite system, that is, $0.61 \AA^{2} / \mathrm{ps}$, which is close to the experimentally observed value, $0.65 \AA^{2} /$ ps. $^{42,48,57,58,62,69,70}$ It is remarkable that the self-diffusion coefficient at 18 
$\mathrm{K}$ has the minimum at the $3: 1$ mixture whose mass is 1.6 times lighter than the pure $\mathrm{D}_{2}$ liquid.(Fig.2(a)) The minimum at the 3:1 mixture rises with increasing the temperature, and the normal mass order is finally recovered at $30 \mathrm{~K}$ as shown in Fig.2(b). The minimum of the self-diffusion coefficient appears since the diffusive dynamics is determined by the balance of the liquid mass and the extent of the supercooling: On one hand, it is general that lighter liquid has a larger self-diffusion coefficient and vice versa. On the other hand, the supercooling reduces a self-diffusion coefficient. These two conflicting effects lead to the current minimum appearing in the self-diffusion coefficients. If any molecules were not supercooled in the mixtures at $18 \mathrm{~K}$, the computed diffusion coefficients at $18 \mathrm{~K}$ would follow the simple mass order as in the $30 \mathrm{~K}$ case. Actually, the difference between the selfdiffusion coefficients of the $\mathrm{H}_{2}$ and $\mathrm{D}_{2}$ components in the mixtures becomes smaller with decreasing the temperature in spite of the twice mass difference, and further the difference between the self-diffusion coefficients of the pure $\mathrm{H}_{2}$ and $\mathrm{D}_{2}$ liquids becomes smaller too reflecting the $\mathrm{H}_{2}$ supercooling at the lower temperature.(See Fig.S6) We note that, as Fig.S6 shows, the diffusion coefficients of each isotope exhibit the similar tendency to the total diffusion coefficients at $18 \mathrm{~K}$. The effectively supercooled $\mathrm{H}_{2}$ molecules decelerate coexisting $\mathrm{D}_{2}$ molecules; slowed down $\mathrm{H}_{2}$ molecules influence and disturb dynamics of coexisting $\mathrm{D}_{2}$ molecules due to the intermolecular interaction between two isotope molecules.

Although the minima of the self-diffusion coefficients were also found in some water solutions, ${ }^{28,29,39-47,71}$ their physical origin is basically a hydrogen-bonded network which does not exist in the present $\mathrm{H}_{2}-\mathrm{D}_{2}$ mixtures. In addition, the simulations of binary systems composed of differently sized model particles reported that the crystal growth becomes slowest for a specific mixing ratio due to the geometric frustration ${ }^{8}$ which again does not happen in the current mixtures; the difference between the H-H and D-D bond length, $0.0149 \AA$ at maximum, is not significant regardless of the $\mathrm{D}_{2}$ mixing ratio, and the tiny geometrical frustration due to the similarly sized molecules does not affect the present self-diffusion coefficients.(See Fig.S4) The fact that only the $25 \%$ mixing of $\mathrm{D}_{2}$ makes the self-diffusion 
coefficient 2.05 times smaller than the self-diffusion coefficient of the pure $\mathrm{H}_{2}$ liquid suggests that there should be an optimal mixing ratio of $\mathrm{H}_{2}$ and $\mathrm{D}_{2}$ to decelerate structural rearrangement from a liquid state to a solid state and hinder the crystallization at low temperature. Such decelerated crystallization dynamics in a supercooled $\mathrm{H}_{2}-\mathrm{D}_{2}$ mixture compared to their pure liquids was actually observed through the Raman spectroscopy. ${ }^{49}$
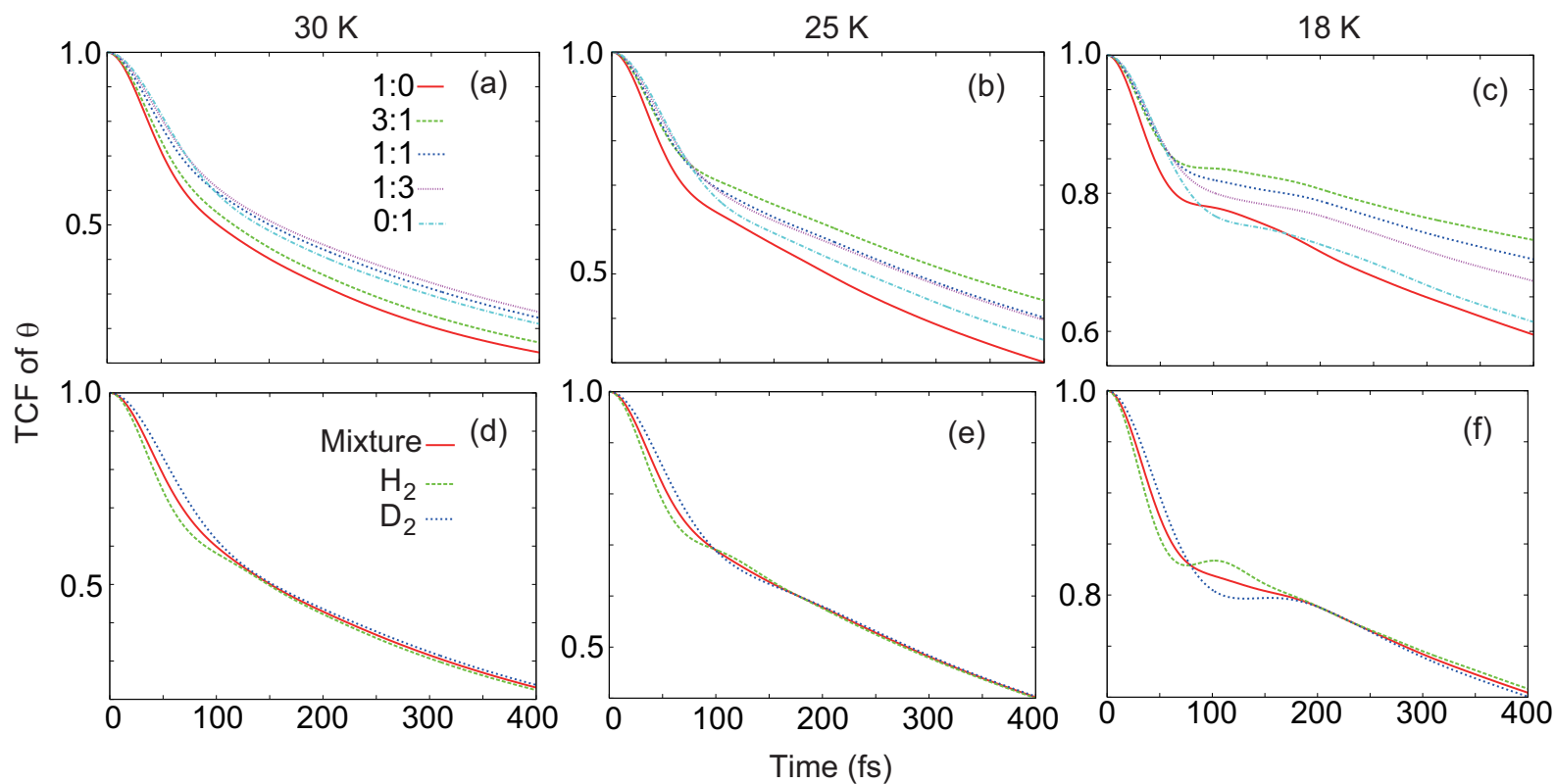

Figure 3: TCFs of the intermolecular angle $\theta(t)$ at (a) $30 \mathrm{~K}$, (b) $25 \mathrm{~K}$ and (c) $18 \mathrm{~K}$. The $\theta$-TCFs decomposed into the $\mathrm{H}_{2}$ and $\mathrm{D}_{2}$ components for the 1:1 mixtures are also shown for (d) $30 \mathrm{~K}$, (e) $25 \mathrm{~K}$ and (f) $18 \mathrm{~K}$.

Angular dynamics occurring in the mixtures, which is ascribed to libration of molecules rather than to rotation, ${ }^{58,60,62}$ can be characterized through the time-correlation functions (TCFs) of the intermolecular angle $\theta(t)$. (Fig.3) The TCF of $\theta(t)$ is defined by $\langle\theta(t) \theta(0)\rangle$ scaled by its initial value at $t=0$ where $\langle\cdots\rangle$ means an ensemble average over different initial times and NWPs. The long-time decay of the $\theta$-TCF reflects the long-range diffusive dynamics, ${ }^{57,58}$ and have the similar decay order to the order of the self-diffusion coefficients shown in Fig.2 except for the pure $\mathrm{D}_{2}$ liquid: e.g. the $\theta$-TCF of the pure $\mathrm{D}_{2}$ liquid at $30 \mathrm{~K}$ decays faster than the $\theta$-TCFs of the lighter $1: 1$ and $1: 3$ mixtures. The $\theta$-TCFs of the pure $\mathrm{D}_{2}$ liquid decay fast because the same diatomic isotope possessing the same mass and shape 
can effectively transfer and exchange its angular momenta. The decay timescales obtained by fitting the long-time $\theta$-TCFs with an exponential function are shown in Fig.S7. They have the maximum at the 3:1 mixture at the lower temperature because of the $\mathrm{H}_{2}$ supercooling just like the minimum of the self-diffusion coefficient at the 3:1 mixture in Fig.2. The present maximum of the decay timescales goes down with increasing the temperature just like the rise of the minimum of the self-diffusion coefficient in Fig.2. The clear bidecay processes appearing in the TCFs of Fig.3 indicate that the qualitatively different two kind of molecular dynamics, the short-time kinetic motion inside a cage and the long-time diffusive dynamics out of a cage, both exist in the mixtures as was reported in the pure $\mathrm{H}_{2}$ and $\mathrm{D}_{2}$ liquid cases. ${ }^{57,60,62}$ Although the $\theta$-TCFs at $25 \mathrm{~K}$ and $18 \mathrm{~K}$ exhibit the clear bidecay process, ${ }^{58,60,62}$ the decay profile of the $\theta$-TCFs at $30 \mathrm{~K}$ seems more likely a single decay process, indicating that a solvation cage is more fragile in the mixtures at $30 \mathrm{~K}$. We note that there is no qualitative difference between the TCFs of $\theta$ and the self-orientation $\phi$ defined in Fig.S8; their angular dynamics is similar and the choice of $\theta$ or $\phi$ does not affect the current conclusions. In Fig.3(d)-(f), the $\theta$-TCFs for the 1:1 mixture are decomposed into the contributions from $\mathrm{H}_{2}$ and $\mathrm{D}_{2}$. At any temperature, the $\theta$-TCFs of $\mathrm{H}_{2}$ initially decay faster than the $\theta$-TCFs of $\mathrm{D}_{2}$ while $\mathrm{H}_{2}$ and $\mathrm{D}_{2}$ complementarily exchange their angular momenta when they are breaking out of a solvation cage as is evidenced by their finally overlapped decay profiles in the long-time region. In other words, the larger angular momenta which lighter $\mathrm{H}_{2}$ initially possess must be transferred to neighboring twice heavier $\mathrm{D}_{2}$ to go out of a solvation cage. The exchange of the angular momenta becomes evident and takes a longer time at the lower temperature due to the stiffer solvation cage and the poorer thermal energy to break the cage. Even the oscillative profiles appear at the lower temperature since the angular momenta exchange is too slow to end in one time. The time to break out of a first solvation cage can be roughly estimated from these exchange times, e.g. 200 fs at $18 \mathrm{~K}$. The general tendency of the other $\theta$-TCFs is quite similar to the 1:1 mixture case except for the ratio-dependent contributions from $\mathrm{H}_{2}$ and $\mathrm{D}_{2}$. (See Fig.S9) 


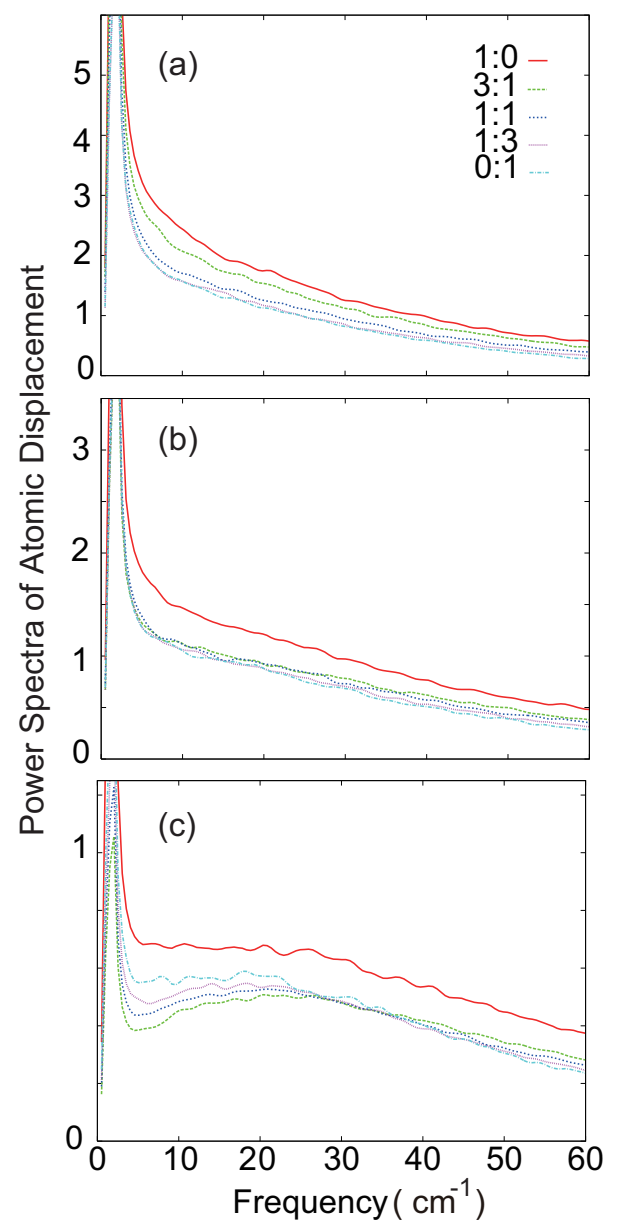

Figure 4: Power spectra of the low-energy collective dynamics at (a) $30 \mathrm{~K}$, (b) $25 \mathrm{~K}$ and (c) $18 \mathrm{~K}$.

Figure 4 displays the power spectra obtained by Fourier-transforming a real-time coordinate displacement of the NWP center in the space-fixed frame, $\left\langle\left|\int d t \sqrt{R_{K}^{2}(t)} \exp (-i \omega t)\right|^{2}\right\rangle$ where $R_{K}(t)$ is the absolute coordinate displacement of the $K$ th NWP center. Such lowfrequency power spectra correspond to the low-energy collective dynamics involved in the mixtures. ${ }^{60,62}$ The power spectra at $30 \mathrm{~K}$ have no peak above $5 \mathrm{~cm}^{-1}$ and just exhibit a simple long tail in the low-frequency region. The power spectra at $25 \mathrm{~K}$ start to exhibit the weak shoulders but still have no evident peak about $5 \mathrm{~cm}^{-1}$. In contrast, the power spectra at $18 \mathrm{~K}$ possess the broad low-frequency peaks around $20 \mathrm{~cm}^{-1}$ which can be assigned as the so-called boson peak that is known as a direct evidence of the supercooled and glassy states. ${ }^{72,73}$ The broad peak around $20 \mathrm{~cm}^{-1}$ also appeared when the pure $\mathrm{H}_{2}$ and $\mathrm{D}_{2}$ liquids 
became supercooled below the freezing temperature, ${ }^{60,62}$ supporting that the mixtures at 18 $\mathrm{K}$ are actually supercooled now. It is interesting that the intensity of the extremely lowfrequency power spectra below $10 \mathrm{~cm}^{-1}$ reflecting the low-energy translational dynamics ${ }^{60,62}$ increases in the same order as the self-diffusion coefficients at any temperature, e.g. in the order of the 1:0, 0:1, 1:3, 1:1, and 3:1 mixtures at $18 \mathrm{~K}$. The extremely low-frequency power spectra stemmed from the long-range diffusive dynamics out of a solvation cage not from the thermal kinetic motion inside a cage corresponding to the initial MSDs in Fig.1(d)-1(f).

In summary, by revealing the interplay between the two isotopes, $\mathrm{H}_{2}$ and $\mathrm{D}_{2}$, in their mixtures, we computationally predicted the significant isotopic mixing effects. Especially, the supercooled $\mathrm{H}_{2}$ lets the self-diffusion coefficient have the minimum at the specific mixing ratio in spite of the little difference in the liquid and molecular structures. The current picture of the component-dependent $\mathrm{H}_{2}$ supercooling is supported by giving the details of what such supercooled state actually entails: (1) The nonlinear MSDs in the initial stage, (2) the similar decay order of the angular dynamics to the anomalous diffusion order, (3) the enhanced bidecay process in the angular TCFs, (4) the broad low-frequency peaks analogous to the boson peaks, and (5) the anomalous order of the spectral intensity of the the extremely low-energy translational dynamics as the self-diffusion coefficient order. It is remarkable that the deceleration of the liquid dynamics was caused by mixing the two isotopes, $\mathrm{H}_{2}$ and $\mathrm{D}_{2}$, which have the same electronic potentials, demonstrating that the mixing itself has a profound impact on the liquid dynamics even without a change of thermodynamic conditions. The above insights provide a physical basis to explain the observed anomalous slowdown of the crystallization in the $\mathrm{H}_{2}-\mathrm{D}_{2}$ quantum mixtures. We propose that there should be an optimal mixing ratio of $\mathrm{H}_{2}$ and $\mathrm{D}_{2}$ to decelerate structural rearrangement and hinder the crystallization. The current high-temperature $\mathrm{H}_{2}$ supercooling induced by the high density also implies the possibility of high-temperature superfluid under high density. The obtained physical insights in this Letter will help in experimentally controlling and achieving unknown isotopic quantum mixtures including superfluid. 


\section{Acknowledgments}

KHD thanks Grant-in-Aids for Scientific Research from Japan Society for the Promotion of Science (KAKENHI), Grant No. 20K05419, Toyota Mobility Foundation and Grant-in-Aid for Scientific Research on Innovative Areas, Grant No.18H05407.

Supporting Information Available. Additional data for structures and dynamics of hydrogen and deuterium mixtures.

\section{References}

(1) Martin, P.-A.; Chen, F.; Forsyth, M.; Deschamps, M.; O’Dell, L. A. Correlating Intermolecular Cross-Relaxation Rates with Distances and Coordination Numbers in Ionic Liquids. J. Phys. Chem. Lett. 2018, 9, 7072-7078.

(2) Banerjee, A.; Chakrabarty, S.; Bhattacharyya, S. M. Interplay between Crystallization and Glass Transition in Binary Lennard-Jones Mixtures. J. Chem. Phys. 2013, 139, 104501.

(3) Han, Y.; Hobbie, E. K.; Kilin, D. S. First-Principles Molecular Dynamics of Monomethylhydrazine and Nitrogen Dioxide. J. Phys. Chem. Lett. 2019, 10, 2394 2399.

(4) Cosby, T.; Kapoor, U.; Shah, J. K.; Sangoro, J. Mesoscale Organization and Dynamics in Binary Ionic Liquid Mixtures. J. Phys. Chem. Lett. 2019, 10, 6274-6280.

(5) Coslovich, D.; Pastore, G. Understanding Fragility in Supercooled Lennard-Jones Mixtures. I. Locally Preferred Structures. J. Chem. Phys. 2007, 127, 124504.

(6) Valdes, L.-C.; Affouard, F.; Descamps, M.; Habasaki, J. Mixing Effects in GlassForming Lennard-Jones Mixtures. J. Chem. Phys. 2009, 130, 154505. 
(7) Pedersen, U. R.; Schroder, T. B.; Dyre, J. C.; Harrowell, P. Geometry of Slow Structural Fluctuations in a Supercooled Binary Alloy. Phys. Rev. Lett. 2010, 104, 105701.

(8) Williams, S. R.; Royall, C. P.; Bryant, G. Crystallization of Dense Binary Hard-Sphere Mixtures with Marginal Size Ratio. Phys. Rev. Lett. 2008, 100, 225502.

(9) Ha, M. Y.; Yoon, T. J.; Tlusty, T.; Jho, Y.; Lee, W. B. Universality, Scaling, and Collapse in Supercritical Fluids. J. Phys. Chem. Lett. 2020, 11, 451-455.

(10) Martinek, T.; Duboué-Dijon, E.; Timr, m.; Mason, P. E.; Baxová, K.; Fischer, H. E.; Schmidt, B.; Pluhařová, E.; Jungwirth, P. Calcium Ions in Aqueous Solutions: Accurate Force Field Description Aided by Ab Initio Molecular Dynamics and Neutron Scattering. J. Chem. Phys. 2018, 148, 222813.

(11) Luzar, A.; Chandler, D. Structure and Hydrogen Bond Dynamics of Water-Dimethyl Sulfoxide Mixtures by Computer Simulations. J. Chem. Phys. 1993, 98, 8160-8173.

(12) Zhang, N.; Shen, Z.; Chen, C.; He, G.; Hao, C. Effect of Hydrogen Bonding on Self-Diffusion in Methanol/Water Liquid Mixtures: A Molecular Dynamics Simulation Study. J. Mol. Liq. 2015, 203, 90-97.

(13) Bakó, I.; Megyes, T.; Bálint, S.; Grósz, T.; Chihaia, V. Water-Methanol Mixtures: Topology of Hydrogen Bonded Network. Phys. Chem. Chem. Phys. 2008, 10, 50045011.

(14) Spickermann, C.; Thar, J.; Lehmann, S. B. C.; Zahn, S.; Hunger, J.; Buchner, R.; Hunt, P. A.; Welton, T.; Kirchner, B. Why are Ionic Liquid Ions Mainly Associated in Water? A Car-Parrinello Study of 1-Ethyl-3-Methyl-Imidazolium Chloride Water Mixture. J. Chem. Phys. 2008, 129, 104505.

(15) Kovacs, H.; Kowalewski, J.; Laaksonen, A. Molecular Dynamics Simulation of Liquid Mixtures of Acetonitrile and Chloroform. J. Phys. Chem. 1990, 94, 7378-7385. 
(16) Megyes, T.; Bálint, S.; Peter, E.; Grósz, T.; Bakó, I.; Krienke, H.; Bellissent-Funel, M.C. Solution Structure of $\mathrm{NaNO}_{3}$ in Water: Diffraction and Molecular Dynamics Simulation Study. J. Phys. Chem. B 2009, 113, 4054-4064.

(17) Dougan, L.; Bates, S. P.; Hargreaves, R.; Fox, J. P.; Crain, J.; Finney, J. L.; Réat, V.; Soper, A. K. Methanol-Water Solutions: A Bi-Percolating Liquid Mixture. J. Chem. Phys. 2004, 121, 6456-6462.

(18) Jiang, W.; Wang, Y.; Voth, G. A. Molecular Dynamics Simulation of Nanostructural Organization in Ionic Liquid/Water Mixtures. J. Phys. Chem. B 2007, 111, 4812-4818.

(19) Das, A. K.; Tembe, B. L. Molecular Dynamics Simulations of Sodium Chloride Solutions in Water-Dimethyl Sulphoxide Mixtures: Potentials of Mean Force and Solvation Structures. J. Chem. Phys. 1999, 111, 7526-7536.

(20) Alavi, S.; Ripmeester, J. A.; Klug, D. D. Molecular Dynamics Simulations of Binary Structure H Hydrogen and Methyl-Tert-Butylether Clathrate Hydrates. J. Chem. Phys. 2009, 124, 204707.

(21) Luque, J.; Santamaría, J.; Freire, J. J. Molecular Dynamics of Chain Molecules in Solution. Static and Dynamic Properties. J. Chem. Phys. 1989, 91, 584-589.

(22) Chowdhuri, S.; Chandra, A. Molecular Dynamics Simulations of Aqueous NaCl and $\mathrm{KCl}$ Solutions: Effects of Ion Concentration on the Single-Particle, Pair, and Collective Dynamical Properties of Ions and Water Molecules. J. Chem. Phys. 2001, 115, 37323741.

(23) Rizzo, R. C.; Jorgensen, W. L. OPLS All-Atom Model for Amines: Resolution of the Amine Hydration Problem. J. Am. Chem. Soc. 1999, 121, 4827-4836.

(24) Borodin, O.; Bedrov, D.; Smith, G. D. Concentration Dependence of Water Dynamics 
in Poly(Ethylene Oxide)/Water Solutions from Molecular Dynamics Simulations. J. Phys. Chem. B 2002, 106, 5194-5199.

(25) Towey, J. J.; Soper, A. K.; Dougan, L. Preference for Isolated Water Molecules in a Concentrated Glycerol-Water Mixture. J. Phys. Chem. B 2011, 115, 7799-7807.

(26) Civera, M.; Fornili, A.; Sironi, M.; Fornili, S. L. Molecular Dynamics Simulation of Aqueous Solutions of Glycine Betaine. Chem. Phys. Lett. 2003, 367, 238-244.

(27) Gratias, R.; Kessler, H. Molecular Dynamics Study on Microheterogeneity and Preferential Solvation in Methanol/Chloroform Mixtures. J. Phys. Chem. B 1998, 102, $2027-2031$.

(28) Overduin, S. D.; Patey, G. N. Comparison of Simulation and Experimental Results for a Model Aqueous Tert-Butanol Solution. J. Chem. Phys. 2017, 147, 024503.

(29) Pereyra, R. G.; Asar, M. L.; Carignano, M. A. The Role of Acetone Dipole Moment in Acetone-Water Mixture. Chem. Phys. Lett. 2011, 507, 240-243.

(30) Mallik, B. S.; Chandra, A. Hydrogen Bond Dynamics and Vibrational Spectral Diffusion in Aqueous Solution of Acetone: A First Principles Molecular Dynamics Study. J. Chem. Sci 2012, 124, 215-221.

(31) Du, H.; Rasaiah, J. C.; Miller, J. D. Structural and Dynamic Properties of Concentrated Alkali Halide Solutions: A Molecular Dynamics Simulation Study. J. Phys. Chem. B 2007, 111, 209-217.

(32) Netz, P. A.; Dorfmüller, T. Computer Simulation Studies on the Polymer-Induced Modification of Water Properties in Polyacrylamide Hydrogels. J. Phys. Chem. B 1998, 102, 4875-4886.

(33) Tamai, Y.; Tanaka, H.; Nakanishi, K. Molecular Dynamics Study of Polymer-Water 
Interaction in Hydrogels. 1. Hydrogen-Bond Structure. Macromolecules 1996, 29, 67506760.

(34) Tamai, Y.; Tanaka, H.; Nakanishi, K. Molecular Dynamics Study of Polymer-Water Interaction in Hydrogels. 2. Hydrogen-Bond Dynamics. Macromolecules 1996, 29, 67616769.

(35) Tamai, Y.; Tanaka, H. Effects of Polymer Chains on Structure and Dynamics of Supercooled Water in Poly(vinyl Alcohol). Phys. Rev. E 1999, 59, 5647-5654.

(36) Corradini, D.; Su, Z.; Stanley, H. E.; Gallo, P. A Molecular Dynamics Study of the Equation of State and the Structure of Supercooled Aqueous Solutions of Methanol. J. Chem. Phys. 2012, 137, 184503.

(37) Smith, G. D.; Bedrov, D. Roles of Enthalpy, Entropy, and Hydrogen Bonding in the Lower Critical Solution Temperature Behavior of Poly(ethylene oxide)/Water Solutions. J. Phys. Chem. B 2003, 10\%, 3095-3097.

(38) Kundu, A.; Dahms, F.; Fingerhut, B. P.; Nibbering, E. T. J.; Pines, E.; Elsaesser, T. Hydrated Excess Protons in Acetonitrile/Water Mixtures: Solvation Species and Ultrafast Proton Motions. J. Phys. Chem. Lett. 2019, 10, 2287-2294.

(39) Derlacki, Z. J.; Easteal, A. J.; Edge, V. J.; Woolf, L. A.; Roksandic, Z. Diffusion Coefficients of Methanol and Water and the Mutual Diffusion Coefficient in MethanolWater Solutions at 278 and 298 K. J. Phys. Chem. 1985, 89, 5318-5322.

(40) Packer, K. J.; Tomlinson, D. J. Nuclear Spin Relaxation and Self-Diffusion in the Binary System, Dimethylsulphoxide(DMSO)+Water. Trans. Faraday Soc. 1971, 67, $1302-1314$.

(41) Dominguez, H.; Pizio, O. On the Composition Dependence of the Microscopic Structure, Thermodynamic, Dynamic and Dielectric Properties of Water-Dimethyl Formamide 
Model Mixtures. Molecular Dynamics Simulation Results. Condens. Matter Phys. 2017, 20, 43602 .

(42) Hone, T. D.; Voth, G. A. A Centroid Molecular Dynamics Study of Liquid ParaHydrogen and ortho-Deuterium. J. Chem. Phys. 2004, 121, 6412-6422.

(43) Pattanayak, S. K.; Chowdhuri, S. Effects of Methanol on the Hydrogen Bonding Structure and Dynamics in Aqueous N-Methylacetamide Solution. J. Mol. Liq. 2014, 194, $141-148$.

(44) Ferrario, M.; Haughney, M.; McDonald, I. R.; Klein, M. L. Molecular-Dynamics Simulation of Aqueous Mixtures: Methanol, Acetone, and Ammonia. J. Chem. Phys. 1990, 93, 5156-5166.

(45) Bouazizi, S.; Nasr, S. Effect of Solvent Composition on the Structural and Dynamical Properties of Sodium Chloride Solutions in Water-Methanol Mixtures. J. Mol. Liq. 2016, 221, 842-850.

(46) Ghoufi, A.; Artzner, F.; Malfreyt, P. Physical Properties and Hydrogen-Bonding Network of Water-Ethanol Mixtures from Molecular Dynamics Simulations. J. Phys. Chem. B 2016, 120, 793-802.

(47) Zhang, N.; Li, W.; Chen, C.; Zuo, J. Molecular Dynamics Simulation of Aggregation in Dimethyl Sulfoxide-Water Binary Mixture. Comput. Theor. Chem. 2013, 1017, 126135.

(48) Souers, P. C. Hydrogen Properties for Fusion Energy; University of California Press, Berkeley, USA, 1986.

(49) Kühnel, M.; Fernández, J. M.; Tramonto, F.; Tejeda, G.; Moreno, E.; Kalinin, A.; Nava, M.; Galli, D. E.; Montero, S.; Grisenti, R. E. Observation of Crystallization 
Slowdown in Supercooled Parahydrogen and Orthodeuterium Quantum Liquid Mixtures. Phys. Rev. B 2014, 89, 180201.

(50) Colognesi, D.; Bafile, U.; Celli, M.; Neumann, M.; Orecchini, A. Hydrogen SelfDynamics in Liquid $\mathrm{H}_{2}-\mathrm{D}_{2}$ Mixtures Studied through Inelastic Neutron Scattering. Phys. Rev. E 2015, 92, 012311.

(51) Kozioziemski, B. J.; Collins, G. W. Raman Spectra of Solid Isotopic Hydrogen Mixtures. Phys. Rev. B 2003, 67, 174101.

(52) White, D.; Gaines, J. R. Liquid-Solid Phase Equilibria in the Hydrogen-Deuterium System. J. Chem. Phys. 1965, 42, 4152-4158.

(53) Galliero, G.; Boned, C. Thermal Conductivity of the Lennard-Jones Chain Fluid Model. Phys. Rev. E 2009, 80, 061202.

(54) Poulsen, J. A.; Nyman, G.; Rossky, P. J. Quantum Diffusion in Liquid Para-Hydrogen: An Application of the Feynman-Kleinert Linearized Path Integral Approximation. $J$. Phys. Chem. B 2004, 108, 19799-19808.

(55) Yonetani, Y.; Kinugawa, K. Transport Properties of Liquid Para-Hydrogen: The Path Integral Centroid Molecular Dynamics Approach. J. Chem. Phys. 2003, 119, 96519660.

(56) Hyeon-Deuk, K.; Ando, K. Intermolecular Diatomic Energies of a Hydrogen Dimer with Non-Born-Oppenheimer Nuclear and Electron Wave Packets. Chem. Phys. Lett. 2012, 532, 124-130.

(57) Hyeon-Deuk, K.; Ando, K. Quantum Molecular Dynamics Simulation of Liquid Para-Hydrogen by Nuclear and Electron Wave Packet Approach. J. Chem. Phys.(Communication) 2014, 140, 171101. 
(58) Hyeon-Deuk, K.; Ando, K. Correlations of Intra- and Intermolecular Dynamics and Structure in Liquid Para-Hydrogen. Phys. Rev. B 2014, 90, 165132.

(59) Hyeon-Deuk, K.; Ando, K. Dynamical and Structural Analyses of Solid Hydrogen under Vapor Pressure. J. Chem. Phys.(Communication) 2015, 140, 171102.

(60) Hyeon-Deuk, K.; Ando, K. Distinct Structural and Dynamical Difference between Supercooled and Normal Liquids of Hydrogen Molecules. Phys. Chem. Chem. Phys. 2016, $18,2314-2318$.

(61) Abe, K.; Hyeon-Deuk, K. Dynamical Ordering of Hydrogen Molecules Induced by Heat Flux. J. Phys. Chem. Lett. 2017, 8, 3595-3600.

(62) Abe, K.; Yamaoka, S.; Hyeon-Deuk, K. Isotopic Effects on Intermolecular and Intramolecular Structure and Dynamics in Hydrogen, Deuterium and Tritium Liquids: Normal Liquid and Weakly and Strongly Cooled Liquids. J. Phys. Chem. B 2018, 122, $8233-8242$.

(63) Silvera, I. F.; Goldman, V. V. The Isotropic Intermolecular Potential for $\mathrm{H}_{2}$ and $\mathrm{D}_{2}$ in the Solid and Gas Phases. J. Chem. Phys. 1978, 69, 4209-4213.

(64) Yonetani, Y.; Kinugawa, K. Centroid Molecular Dynamics Approach to the Transport Properties of Liquid Para-Hydrogen over the Wide Temperature Range. J. Chem. Phys. 2004, 120, 10624-10633.

(65) Geneste, G.; Torrent, M.; Bottin, F.; Loubeyre, P. Strong Isotope Effect in Phase II of Dense Solid Hydrogen and Deuterium. Phys. Rev. Lett. 2012, 109, 155303.

(66) Helfferich, J.; Ziebert, F.; Frey, S.; Meyer, H.; Farago, J.; Blumen, A.; Baschnagel, J. Continuous-Time Random-Walk Approach to Supercooled Liquids. I. Different Definitions of Particle Jumps and Their Consequences. Phys. Rev. E 2014, 89, 042603. 
(67) Helfferich, J.; Ziebert, F.; Frey, S.; Meyer, H.; Farago, J.; Blumen, A.; Baschnagel, J. Continuous-Time Random-Walk Approach to Supercooled Liquids. II. Mean-Square Displacements in Polymer Melts. Phys. Rev. E 2014, 89, 042604.

(68) Ngai, K. L.; Paluch, M. Isotopic Effects on Intermolecular and Intramolecular Structure and Dynamics in Hydrogen, Deuterium and Tritium Liquids: Normal Liquid and Weakly and Strongly Cooled Liquids. J. Chem. Phys. 2004, 120, 857-873.

(69) OReilly, D. E.; Peterson, E. M. Self-Diffusion of Liquid Hydrogen and Deuterium. J. Chem. Phys. 1977, 66, 934-937.

(70) Georgescu, I.; Deckman, J.; Fredrickson, L. J.; Mandelshtam, V. A. Thermal Gaussian Molecular Dynamics for Quantum Dynamics Simulations of Many-Body Systems: Application to Liquid Para-Hydrogen. J. Chem. Phys. 2011, 134, 174109.

(71) Aparicio, S.; Alcalde, R.; Trenzado, J. L.; Caro, M. N.; Atilhan, M. Study of Dimethoxyethane/Ethanol Solutions. J. Phys. Chem. B 2011, 115, 8864-8874.

(72) Yamamuro, O.; Madokoro, Y.; Yamasaki, H.; Matsuo, T.; Tsukushi, I.; Takeda, K. Low-Energy Excitations of Vapor-Deposited Amorphous Ice and Its Annealing and Methanol-Doping Effects Studied by Inelastic Neutron Scattering. J. Chem. Phys. 2001, 115, 9808-9814.

(73) Kanaya, T.; Kawaguchi, T.; Kaji, K. Low-Energy Excitation and Fast Motion near T in Amorphous cis1,4-polybutadiene. J. Chem. Phys. 1993, 98, 8262-8270. 